\title{
Manifestaciones neuro-psiquiátricas en una paciente con traumatismo encéfalo-craneano.
}

\author{
Neuropsychiatric manifestations in a patient with traumatic brain injury. \\ Nataly Chirinos-Montes ${ }^{1}$, Débora Contreras-Toledo ${ }^{1}$, Antonio Lozano-Vargas ${ }^{2}$
}

\section{RESUMEN}

Presentamos el caso de una mujer de 18 años de edad quien luego de sufrir un traumatismo encéfalo-craneano severo exacerba sus rasgos impulsivos de personalidad y desarrolla desinhibición sexual, agresividad, perseveración, delusiones, amnesia, síndrome depresivo y maniforme.

PALABRAS CLAVE: Traumatismo encéfalo-craneano, personalidad orgánica, amnesia, depresión, manía.

\section{SUMMARY}

We present a case of an 18-year-old woman who after a severe traumatic brain injury showed an exacerbation of her impulsivity traits and developed sexual desinhibition, aggressiveness, perseveration, delusions, amnesia, and depressive and maniac syndromes.

KEYWORDS: Traumatic brain injury, organic personality, amnesia, depression, mania.

\section{INTRODUCCIÓN}

El traumatismo encéfalo craneano (TEC) es la primera causa de muerte y de discapacidad en la población menor de 45 años de edad, siendo la mayor incidencia entre los 15 y los 25 años.

Las causas de TEC más importantes son los accidentes automovilísticos y de trabajo, destacando la caída de altura (34\%), atropellos (31\%), choques (24\%) y golpes directos (10\%) (1). Los pacientes que sufren un daño orgánico-cerebral post TEC presentan secuelas emocionales y aproximadamente el $40 \%$ desarrollan más de un desorden psiquiátrico; en donde los cambios físicos, conductuales o emocionales dependen del área cerebral que ha sido afectada $(1,2)$. Los cambios en la personalidad se caracterizan por agresividad, desinhibición, labilidad emocional o acentuación de los rasgos de personalidad premórbida (2). Algunos estudios han demostrado que la presencia de agresividad luego de sufrir un TEC se correlaciona con depresión, con lesión del lóbulo frontal, con un pobre funcionamiento psicosocial previo al TEC y con una historia de abuso de alcohol y otras drogas (3). En el presente artículo se presenta el caso de una paciente joven que desarrolló un trastorno orgánico-cerebral con cambios en su comportamiento luego de sufrir un TEC que comprometió diversas áreas funcionales cerebrales.

\section{Caso clínico}

La paciente es una mujer de 18 años de edad, conviviente, evangélica, con secundaria

1 Estudiante de Medicina. Facultad de Medicina Alberto Hurtado. Universidad Peruana Cayetano Heredia. Lima, Perú.

2 Médico Psiquiatra. Hospital Nacional Cayetano Heredia. Profesor de la Universidad Peruana Cayetano Heredia. Lima, Perú. 
completa; quien hace 14 meses sufrió un accidente automovilístico estando en compañía de su hija de 8 meses de edad. El accidente se produjo por exceso de velocidad al impactar el automóvil contra un muro de cemento. Como consecuencia del accidente, la paciente sufrió un TEC severo, fractura de tibia y peroné de la pierna derecha, y fractura del tabique nasal y del maxilar superior derecho. Llegó a la emergencia de un hospital general con compromiso de la conciencia y hemodinámicamente inestable con presión arterial de 85/60 $\mathrm{mm} \mathrm{Hg}$, frecuencia cardiaca de más de 100 por minuto y frecuencia respiratoria de 26 por minuto; ingresando a la unidad de cuidados intensivos para soporte inotrópico, ventilatorio y monitoreo neuro-intensivo. En dicha unidad le indujeron un coma barbitúrico debido al aumento de la presión intracraneana manteniéndose posteriormente con un Glasgow en 7.

Durante su hospitalización se le realizaron diversos procedimientos como una traqueostomía para ventilación endotraqueal y la colocación de un catéter venoso central, sonda uretral, sonda nasogástrica y vías periféricas de acceso venoso. La paciente desarrolló una neumonía intrahospitalaria y recibió cobertura antibiótica.

En su evolución presentó un paro cardiorespiratorio por lo cual recibió maniobras de resucitación recuperando los signos vitales luego de aproximadamente 8 minutos. Además, mantuvo un Glasgow en 8 y durante muchos días su evolución fue estacionaria.

Hace 13 meses la paciente fue recuperando su estado de conciencia y su salud física por lo cual fue hospitalizada en una sala de medicina donde completó su recuperación y fue dada de alta luego de aproximadamente 30 días. Hace 12 meses comenzó a comunicarse escribiendo en un papel, no reconociendo a su madre escribiendo "Tú no eres mi madre, seguro me has adoptado". Desde aquel momento la paciente empezó a manifestar alteraciones de la memoria caracterizadas por tener dificultad para retener información en su interacción familiar cotidiana, incluso cada vez que visitaba a su médico manifestaba no recordarlo, otras veces lo confundía con otra persona o experimentaba la consulta médica como si fuera la primera vez. Además, tenía la dificultad de recordar algunos hechos significativos vividos previamente $\mathrm{y}$, por ejemplo, a pesar que se esforzaba por recordar lo que había ocurrido el día del accidente, no lograba evocarlo. Asimismo, al intentar recordar un hecho del pasado alteraba el contenido y lo narraba como si hubiera ocurrido de otro modo; otras veces, al imaginarse un determinado hecho lo experimentaba como si fuera un recuerdo real, como si le hubiera ocurrido realmente.

Además, presentaba ánimo expansivo; manifestaba que sus familiares le querían robar sus pertenencias; se distraía fácilmente, pasaba de un tema a otro con gran facilidad, a veces regresaba al punto inicial de la conversación y otras veces se dispersaba; perseveraba en el tema de su suegra; y su lenguaje era abundante en su contenido y muchas veces soez.

Desde hace 9 meses, la paciente desarrolló cambios en su comportamiento, mostrándose irritable, agresiva, desinhibida, impulsiva y con pérdida de interés en el cuidado de su aliño personal; es así que la mantuvieron encerrada en su habitación por temor a que saliera de casa y se perdiera; tal cual había ocurrido en dos oportunidades en las que había tomado el autobús equivocado y no supo cómo retornar a casa.

La paciente reaccionaba violentamente con insultos y con agresión física cada vez que algún transeúnte en la vía pública tropezaba con ella de forma incidental o cuando recibía un cumplido o piropo. Además, en una oportunidad tuvo una discusión con su suegra a quien tomó del cabello y golpeó su cabeza tres veces contra la pared. Por otra parte, la actitud desinhibida se manifestaba cada vez que se acercaba a entablar comunicación con desconocidos en cuya interacción exhibía excesiva confianza y traslucía cierto erotismo hacia los interlocutores masculinos. Por otro lado, su irritabilidad aumentó de forma especial en su interacción con su suegra y su pareja; haciéndose además muy notoria su desvinculación afectiva con su menor hija.

Hace 8 meses fue evaluada por el servicio de psiquiatría por primera vez, manifestando falta de voluntad, tristeza, sentimientos de frustración, irritabilidad, llanto e ideas pasivas de muerte siendo medicada en un primer momento con sertralina 50 $\mathrm{mg}$ /día lo cual aparentemente desencadenó mayor irritabilidad, tendencia a la agresividad y síntomas maniformes por lo cual posteriormente fue suspendida y reemplazada por valproato de sodio $1000 \mathrm{mg}$ /día con titulación progresiva. Sin embargo, la paciente recibía la medicación de manera irregular ya que empezó a observar aumento de peso por lo cual suspendió la medicación. Esta mala adherencia al tratamiento fue consecuencia además de un pobre soporte y 
compromiso familiar.

Como antecedentes, la paciente creció en un ambiente familiar disfuncional, su padre consumía drogas y no lo conoció sino hasta los 18 años de edad y de manera anecdótica el mismo día y horas antes del accidente. La paciente no tuvo una figura paterna que acompañara su proceso de crecimiento y su madre no desarrolló un vínculo sano con la paciente siendo criada por los abuelos maternos. A los 12 años de edad empezó a desafiar las reglas del hogar reaccionando con agresividad hacia su madre cuando le prohibía las salidas. En una oportunidad intentó incluso patear el abdomen de su madre cuando ésta se encontraba gestando. En relación a su desarrollo académico, la paciente fue una buena alumna en los primeros años escolares; sin embargo, en la secundaria empezó a presentar problemas de conducta por lo que estuvo con matrícula escolar condicional en dos colegios diferentes. La paciente inició su vida sentimental y sexual muy tempranamente quedando embarazada a los 16 años de edad de una pareja que le duplicaba la edad. La convivencia con su pareja fue inestable, discutían con frecuencia y en repetidas oportunidades la paciente abandonó el hogar.
Al examen físico, la paciente presentaba una constitución pícnica con un índice de masa corporal de 31, dos tatuajes: uno de $2 \times 2 \mathrm{~cm}$ en la muñeca derecha con las iniciales de su nombre y la de su pareja actual, y el otro en el hombro izquierdo de 7 x $3 \mathrm{~cm}$; además se halló un reflejo palmo mentoniano positivo. En el examen mental gesticulaba constantemente, presentaba presión del habla, se anticipaba a las preguntas formuladas, su lenguaje era tangencial y coprolálico, tocaba temas ajenos a los propuestos y había pobreza en el análisis de sus ideas. Presentaba puerilidad, desinhibición, tendencia a la distraibilidad, impulsividad, afecto expansivo, asociaciones por asonancia, y desorientación por momentos en tiempo y espacio. Era verborreica y perseverante en el tema de "su suegra", luego se agotaba y presentaba ansiedad al no poder continuar respondiendo a su interlocutor. En relación a la evolución, se decidió cambiar la medicación a carbamazepina $400 \mathrm{mg} /$ día con titulación progresiva presentando una buena tolerancia, añadiéndose posteriormente sulpirida $200 \mathrm{mg} /$ día con lo cual mejoró su regulación afectiva e impulsividad. Se decidió además completar sus estudios con un electroencefalograma, pruebas neuropsicológicas, control de hemograma y dosaje de carbamazepina. Se
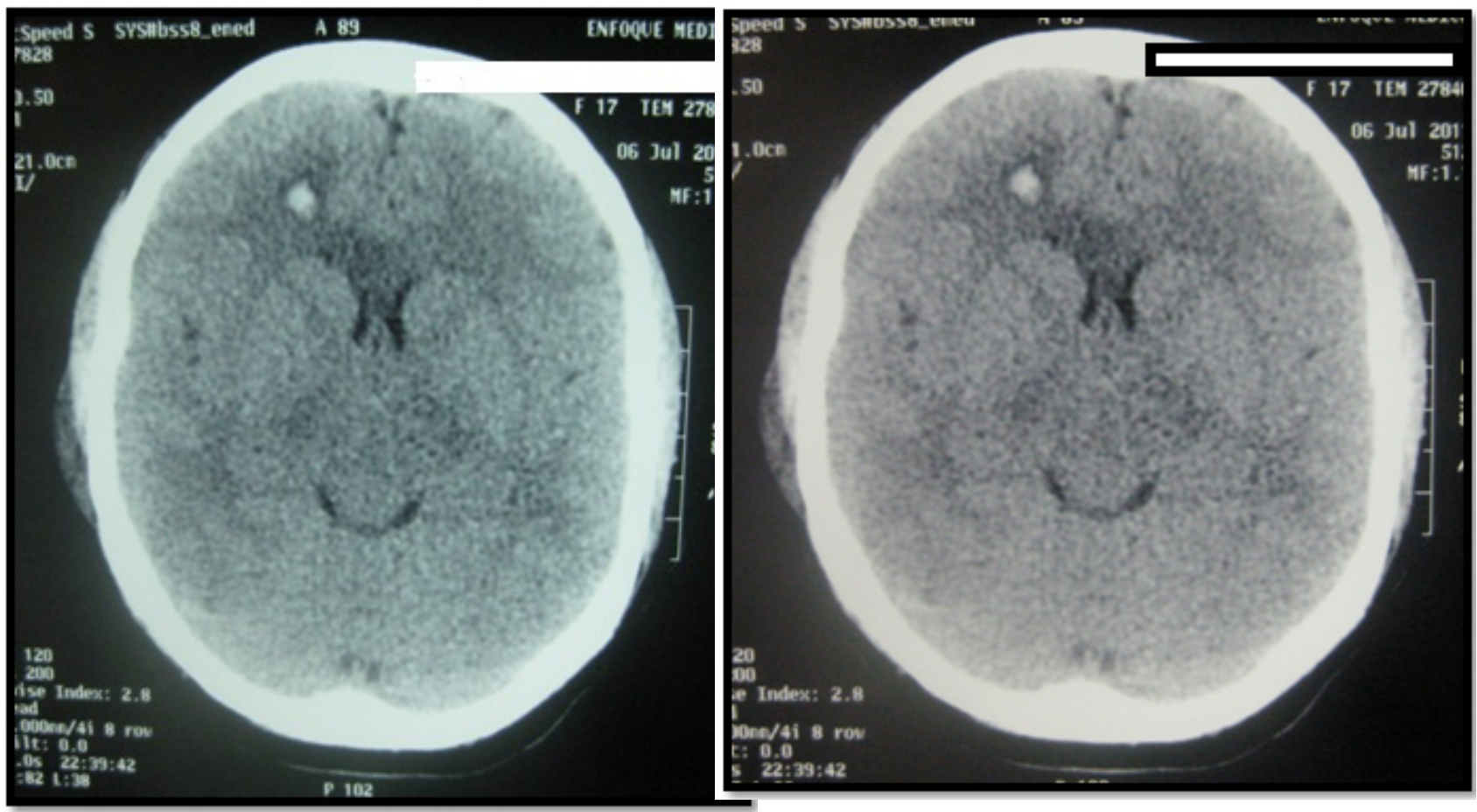

Figura 1. Tomografía de encéfalo: No se observan los ventriculos bien definidos lo que nos indica un cerebro edematizado con una zona radiopaca producida por el sangrado en zona frontal derecha. 


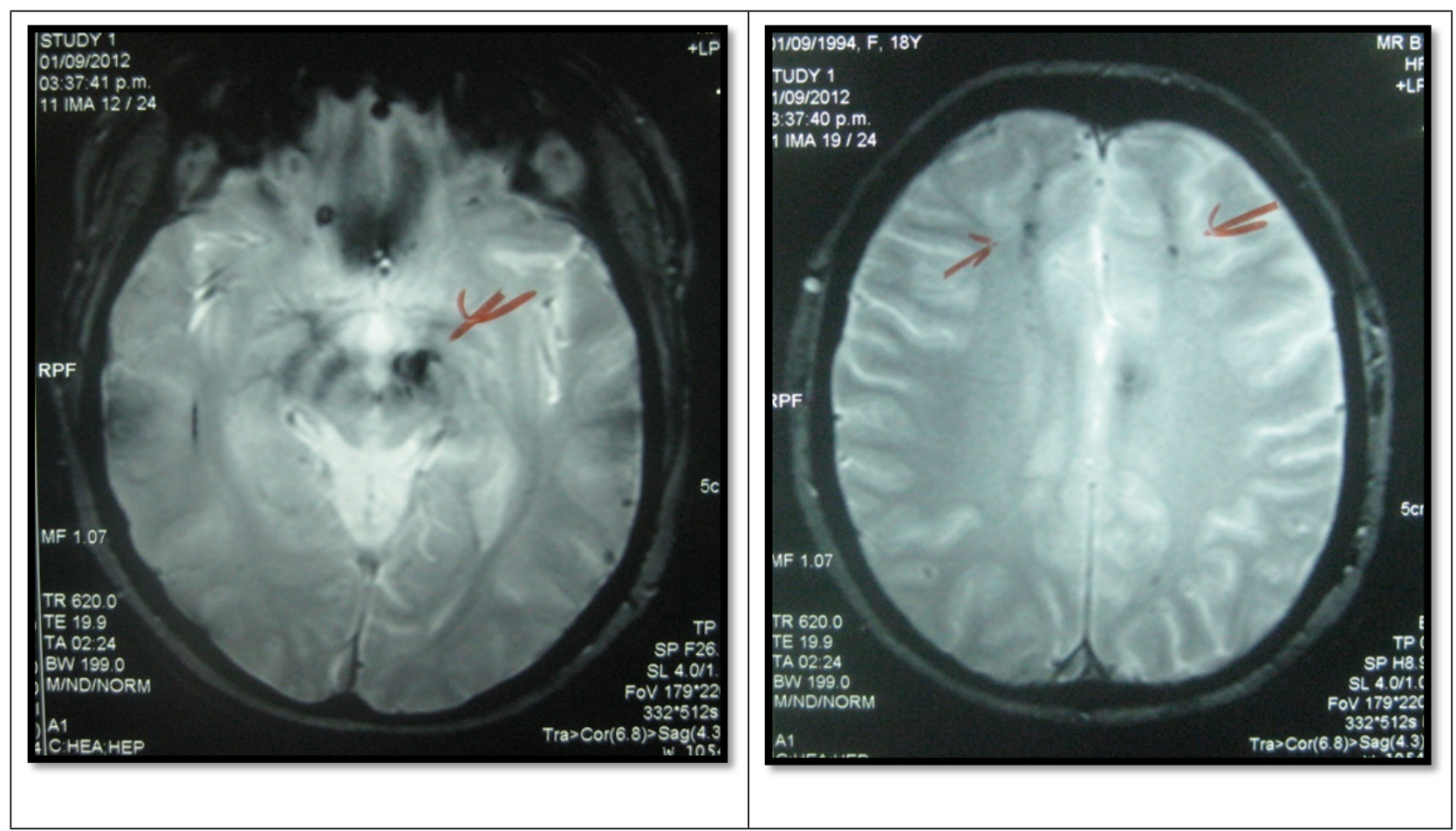

Figura 2: Resonancia magnética: las zonas hipointensas representan areas de multiples sangrados, estos representan depositos de hemosiderina que se observan tanto en region temporal como frontal. Se observa un cerebro menos edematizado a comparacion de la TAC del 06/07/2011.

inició su proceso de rehabilitación y se puso énfasis en mejorar el compromiso y la adherencia al tratamiento por parte de la familia.

\section{Exámenes auxiliares}

Dos tomografías cerebrales sin contraste (Figura 1) muestran: la primera, una contusión hemorrágica frontal derecha y edema cerebral difuso; y la segunda, una contusión cerebral con componente hemorrágico a nivel frontal derecho. Posteriormente, una resonancia magnética de encéfalo sin contraste (Figura 2) evidencia restos hemáticos por lesión axonal difusa, supra e infra tentorial así como restos de sangrado antiguo subaracnoideo.

\section{DISCUSIÓN}

En la literatura científica se describe que los procesos biomecánicos y citotóxicos inducidos por un TEC severo afectan la cara anterior y ventral del lóbulo frontal, del lóbulo temporal medial, el diencéfalo, el tronco cerebral superior y la sustancia blanca. Estas son áreas neuro-conductuales importantes y las lesiones traumáticas en dichas áreas generan perturbaciones en el comportamiento. Así, los pacientes que han sufrido
TEC moderado o severo muestran alteraciones neuro-conductuales que son congruentes con las lesiones cerebrales y generalmente evolucionan de manera predecible (4). La presentación clínica neuroconductual luego de un TEC incluye: coma, delirio, deterioro de la excitación con o sin agitación, amnesia, deterioro en el aprendizaje, alteración en la atención, en la memoria y en otras funciones cognitivas (4).

Alrededor del 32\% de los pacientes que sufren un daño orgánico cerebral post TEC presentan secuelas emocionales que cumplen criterios para el diagnóstico de trastorno orgánico de la personalidad incorporados en la Clasificación Internacional de Enfermedades de la OMS (CIE-10). Según esta clasificación, para hacer el diagnóstico se deben presentar dos o más de los siguientes rasgos: a) capacidad permanentemente reducida para mantener una actividad orientada a un fin, b) alteraciones emocionales como labilidad emocional, simpatía superficial, cambios rápidos hacia la irritabilidad, manifestaciones de agresividad y apatía, c) expresión de impulsos sin considerar las consecuencias sociales como actos antisociales, comportamiento sexual inadecuado o descuido en el aseo personal, d) alteración cognoscitiva, suspicacia, ideas paranoides o preocupación excesiva en un tema 
único, e) alteración en el ritmo y flujo del lenguaje como circunstancialidad, pegajosidad o hipergrafía o f) alteración del comportamiento sexual (3).

En un estudio realizado en el Reino Unido con 164 pacientes después de un año de sufrir un TEC, se encontró que el $21,3 \%$ de la muestra recibió un diagnóstico psiquiátrico, 13,9\% fue diagnosticado de depresión y $9 \%$ de ataque de pánico; cifras que fueron significativamente mayores que en la población general (5). Asimismo, en el año 2002, en un trabajo realizado en Finlandia se observa que el $48 \%$ de pacientes que tuvieron un TEC presentaron trastornos psiquiátricos, siendo el diagnóstico más común la depresión mayor (26,7\%) (5). Mientras que en el año 2010, un estudio en Australia realizado con 1084 pacientes hospitalizados por TEC mostró que doce meses después el 31\% de los pacientes presentaba un trastorno psiquiátrico y el $22 \%$ desarrolló un trastorno psiquiátrico que nunca había experimentado. Los trastornos psiquiátricos más comunes fueron depresión (9\%), trastorno de ansiedad generalizada $(9 \%)$, trastorno de estrés postraumático (TEPT) $(6 \%)$ y agorafobia (6\%) (6). De esta manera, la mayoría de estudios han prestado atención a la incidencia de TEPT y depresión después de un TEC; así los estudios indican que de 10 a $20 \%$ de los sobrevivientes de lesiones traumáticas desarrollan TEPT $(7,8)$ y de 9 a $15 \%$ desarrollan un trastorno depresivo mayor $(7,9)$. Por otra parte, algunos estudios sugieren mayores tasas de trastornos por uso de sustancias y de ansiedad después de un TEC $(7,10)$, pero la mayoría de los estudios indican que los trastornos psiquiátricos después de un trauma son típicamente comórbidos con el trastorno de estrés postraumático (11).

En otros estudios, los trastornos del ánimo después de un TEC se observan con una frecuencia mayor que en la población general con estimaciones que se acercan a $25-50 \%$ para depresión mayor, $15-30 \%$ para distimia y $9 \%$ para manía (12). Así, un estudio que evaluó durante 12 meses a los pacientes que sufrieron un TEC determinó la presencia de manía con una frecuencia del 9\% (13) y este valor fue significativamente mayor que el observado en otras poblaciones con otras lesiones cerebrales como por ejemplo los pacientes con accidente cerebro vascular (14). Se han descrito además posibles factores predisponentes para el desarrollo de manía después de un TEC y que incluyen daño cerebral en la región basal del lóbulo temporal derecho y en la corteza orbitofrontal derecha en pacientes que tienen antecedentes familiares de trastorno bipolar (15).
Así, en las neuro-imágenes de nuestra paciente observamos un daño difuso cerebral supra e infra tentorial. El daño difuso se produce en los casos en que el impacto provoca que el cerebro se mueva hacia delante y hacia atrás dentro del cráneo, conocido como mecanismo del latigazo. Los lóbulos frontal y temporal son los más afectados por este proceso ya que se ubican en cavidades del cráneo donde hay muy poco espacio para amortiguar el daño. Las alteraciones ocasionadas por este daño varían de persona a persona dependiendo de muchos factores, tales como la estructura de la personalidad, la condición premórbida y la severidad del daño cerebral $(3,5)$.

De acuerdo a los criterios clínicos para el diagnóstico de trastorno orgánico de la personalidad, la paciente presenta manifestaciones de irritabilidad, impulsividad, agresividad, suspicacia, ideas paranoides, lenguaje circunstancial y desinhibición sexual. Además, la paciente presentaba alteraciones en la memoria caracterizadas por amnesia anterógrada de forma parcial al tener dificultad para fijar información en su interacción familiar y social cotidiana o cada vez que visitaba a su médico; alteración de la memoria retrógrada de tipo cualitativo evidenciada en el falso reconocimiento (cuando confundía a su médico con otra persona), en la ilusión del recuerdo o alomnesia (al intentar recordar un hecho del pasado alteraba el contenido) y en la paramnesia fantástica (al imaginarse un determinado hecho lo experimentaba como si le hubiera ocurrido realmente); y alteración de la memoria retrógrada de tipo cuantitativo reflejado en la amnesia lacunar al tener dificultad de evocar hechos significativos vividos previamente.

Asimismo, tenía alteración del pensamiento al presentar delusiones de daño; alteración de la voluntad al cursar con perseveración y verbigeración; alteración de la atención al presentar inestabilidad y fácil fatiga de la atención; alteración del lenguaje al presentar tangencialidad en su discurso, verborrea y coprolalia; alteración de la regulación de las emociones al cursar con labilidad emocional, agresividad, desinhibición e impulsividad; así como un cortejo de síntomas correspondientes a un síndrome depresivo $\mathrm{y}$ maniforme. A todo ello podríamos añadir que si bien la paciente presentaba previamente rasgos inestables e impulsivos de personalidad, el daño orgánico cerebral exacerbó dichos rasgos. Por otro lado, la acentuación de la sintomatología irritable y la conducta maniforme a partir del uso de un antidepresivo hace sospechar de un viraje al polo afectivo maniaco por lo cual al añadir un estabilizador del ánimo la sintomatología 
maniforme mejoró. Asimismo, la presencia del reflejo palmo mentoniano pone de manifiesto un posible daño a nivel del lóbulo frontal, siendo la lesión a este nivel el origen de los cambios en la conducta manifestada principalmente con desinhibición e impulsividad.

Finalmente, la edad de la paciente le confiere aparentemente un buen pronóstico, sin embargo, la severidad del TEC, la personalidad previa de la paciente y la pobre adherencia y compromiso familiar al tratamiento no favorecen una adecuada rehabilitación.

\section{Correspondencia:}

Antonio Lozano Vargas

Jr. Puente y Cortez 599

Magdalena del Mar. Lima, Peru.

Correo electrónico: antoniolv2000@yahoo.com

\section{REFERENCIAS BIBLIOGRÁFICAS}

1. Maturana R, Maturana R. Algunos factores predictivos en la evolución del daño orgánico cerebral post traumatismo encéfalo craneano. Ciencia y Trabajo. 2007; 24: 69-75.

2. Vaishnavi S, Rao V, Fann JR. Neuropsychiatric problems after traumatic brain injury: Unraveling the silent epidemic. Psychosomatics. 2009; 50:198-205.

3. Rao V, Rosenberg P, Bertrand M, Salehinia S, Spiro J, Vaishnavi S, et al. Aggression after traumatic brain injury: Prevalence and correlates. J Neuropsychiatry Clin Neurosci. 2009; 21:420-429.

4. Arciniegas DB, McAllister TW. Neurobehavioral management of traumatic brain injury in the critical care setting. Crit Care Clin. 2008; 24:737-765.

5. Schwarzbold, Diaz A, Tostes E, et al. Psychiatric disorders and traumatic brain injury. Neuropsychiatr Dis Treat. 2008: 4: 797-816.
6. Bryant RA, Creamer M, O'Donnell M, Silove D, Clark CR, McFarlane AC. The psychiatric sequelae of traumatic Injury. Am J Psychiatry. 2010; 167:312320.

7. O'Donnell ML, Creamer M, Pattison P, Atkin C. Psychiatric morbidity following injury. Am J Psychiatry. 2004; 161:507-514.

8. Zatzick DF, Jurkovich GJ, Gentinello D, Wisner D, Rivara FP. Posttraumatic stress, problem drinking and functional outcomes after injury. Arch Surg. 2002; 137: 200-205.

9. Shalev AY, Freedman S, Peri T, Brandes D, Sahar T, Orr SP, Pitman RK. Prospective study of posttraumatic stress disorder and depression following trauma. Am J Psychiatry. 1998; 155:630-637.

10. Mayou R, Bryant B, Ehlers A. Prediction of psychological outcomes one year after a motor vehicle accident. Am J Psychiatry. 2001; 158:12311238.

11. North CS, Nixon SJ, Shariat S, Mallonee S, McMillen JC, Spitznagel EL, et al. Psychiatric disorders among survivors of the Oklahoma City bombing. JAMA. 1999; 282:755-762.

12. Taylor CA, Jung HY. Disorders of mood after traumatic brain injury. Semin Clin Neuropsychiatry. 1998;3: 224-231.

13. Jorge RE, Robinson RG, Starkstein SE, Arndt SV, Forrester AW, Geisler FH. Secondary mania following traumatic brain injury. Am J Psychiatry. 1993; 150: 916-921.

14. Van Reekum R, Bolago I, Finlayson MA, Garner S, Links PS. Psychiatric disorders after traumatic brain injury. Brain Inj. 1996; 10:319-327.

15. Silver JM, Hales, RE, Kupfer DJ. Neuropsychiatric aspects of traumatic brain injury. En: Yudofsky SC, Hales RE. (Edit). The American psychiatric press textbook of neuropsychiatry and behavioral neurosciences. Third edition. Arlington: American Psychiatric Publishing Inc; 1997. p. 521-561. 